\title{
Métodos de investigación in vitro de los factores que afectan la durabilidad de la adhesión a dentina
}

\section{In vitro investigation's methods of the factors influencing resin-dentin bond durability}

\author{
Albaladejo A*
}

\section{RESUMEN}

La durabilidad de la adhesión resina-dentina es de crucial importancia tanto para el clínico como para el investigador, sin embargo, se sabe muy poco acerca de la estabilidad de la capa híbrida. El test de fuerzas de adhesión inmediata no puede demostrar adecuadamente los efectos que pueden tener en la durabilidad de la adhesión los poros y otros defectos internos producidos en la capa híbrida. Después del ciclado mecánico, el efecto de estas irregularidades interfaciales que alteran la durabilidad de la adhesión puede resultar más aparente. El uso combinado del ciclado mecánico con el test de fuerzas adhesivas de microtensión permite la evaluación in vitro de la durabilidad de la adhesión resina-dentina bajo unas condiciones clínicamente más relevantes de las que son usadas normalmente en la técnica de fuerzas de adhesión estáticas. El método de degradación basado en la inmersión de los especímenes en hipoclorito de sodio acuoso durante un corto periodo de tiempo resulta bastante más real que los estudios in vitro realizados con almacenamiento en agua durante un largo periodo de tiempo. La disminución de los valores de las fuerzas de adhesión obtenidas después de la inmersión es similar a los obtenidos con estudios de degradación in vivo.

Palabras clave: Dentina, degradación, adhesión, ciclado mecánico, hipoclorito de sodio.

\section{SUMMARY}

The durability of resin-dentin bonds between adhesive resins and coronal dentin is of critical importance and little is known regarding the stability of hybridized layers. Static bond strength tests may not adequately demonstrate the potential detrimental effects that porosities and other internal defects within the adhesive layer may have on bonding durability. After cyclic loading, the effect of these interfacial defects on long-term bonding may be more readily apparent. The combined use of mechanical loading with microtensile bond strength testing permits the evaluation of the in vitro durability of resin-dentin bonds under more clinically-relevant conditions than are usually employed in static bond strength testing techniques. The challenging method based upon $10 \%$ Sodium hypochlorite immersion of specimens during a short period of time is much more reliable than in vitro studies based on long-term water storage of specimens and those previously reported after Sodium hypochlorite immersion are similar to the decline in bond strength obtained when in vivo degradation studies are performed.

Key words: Dentin, resin, degradation, bonding, cyclic loading, sodium hypochlorite.

Fecha de recepción: Junio 2007.

Aceptado para publicación: Noviembre 2007.

* Profesor Asociado de Ortodoncia. Facultad de Odontología. Universidad de Salamanca.

Albaladejo A. Métodos de investigación in vitro de los factores que afectan la durabilidad de la adhesión a dentina. Av. Odontoestomatol 2008; 24 (4): 267-276. 


\section{ADHESIÓN}

La palabra adhesión proviene del latín ad y haerere y significa unir a (1).

La dentina puede ser descrita como un composite biológico, con un relleno mineral de cristales de hidroxiapatita y una matriz formada por una red de fibras de colágeno (2), así, el mecanismo básico de adhesión a dentina es esencialmente un proceso de intercambio que envuelve el reemplazamiento de minerales removidos de los tejidos dentales duros por monómeros de resina que producen un cierre micromecánico en las porosidades creadas (3-5). Este cierre fue descrito por primera vez por Nakabayashi y cols. (6) y es referido comúnmente como "hibridación" o la formación de la capa híbrida.

\section{Adhesión a dentina}

Al comienzo del desarrollo de la Odontología Adhesiva se buscó una adhesión química al calcio o a las fibras de colágena de la estructura dentaria. Hoy en día se habla sobre todo de adhesión mecánica o, más concretamente, adhesión micromecánica $(7,8)$.

La adhesión micromecánica a la dentina está basada en tres mecanismos (9): 1) una adhesión mediante la infiltración de la dentina intertubular y la formación de la capa híbrida o zona de interdifusión. El mecanismo por el que se forma la citada capa, consiste en la infiltración de un monómero adhesivo en la dentina descalcificada con las fibras de colágena expuestas que, tras polimerizar, queda entremezclado con la estructura dental desmineralizada $(7,10)$; 2) una adhesión mediante la infiltración de los túbulos dentinarios y sus ramas laterales, los cuales, tras ser abiertos con el grabado ácido, se produce una infiltración de resina debido al fenómeno de capilaridad, formándose los tags de resina (11) y; 3) una adhesión superficial, por el contacto entre el adhesivo y el sustrato dentinario (12).

\section{Sistemas adhesivos}

Los adhesivos dentinarios se pueden clasificar atendiendo a numerosos criterios. Hoy en día, la manera más clara de clasificar los adhesivos, es la establecida por Van Meerbeek y cols. (8), los cuales establecieron una clasificación según el mecanismo de acción y el número de pasos empleados. De esta manera, podemos encontrar: 1) adhesivos que eliminan el barrillo dentinario, también conocidos como sistemas de grabado total, que acondicionan la dentina con un ácido que remueve totalmente el barrillo dentinario (8); 2) adhesivos que disuelven el barrillo dentinario, los cuales, son sistemas adhesivos que mezclan de forma homogénea el barrillo con el primer y simultáneamente desmineralizan la superficie del sustrato (8). Como no son lavados, el barrillo se incorpora al proceso de adhesión reduciéndose los problemas asociados a la sensibilidad de la técnica $(13,14)$ y; 3) los adhesivos que modifican el barrillo dentinario haciéndolo más poroso para que la resina acceda a la dentina subyacente (12).

\section{DEGRADACIÓN DE LA INTERFASE ADHESIVA IN VITRO Y SUS MÉTODOS DE INVESTIGACIÓN}

Es bastante difícil desarrollar condiciones de laboratorio que puedan testar la longevidad de la adhesión debida a los numerosos factores envueltos en la degradación de la adhesión y a que el desarrollo oral es dinámico y biológicamente complejo (15). Se han presentado diversos métodos para reproducir una situación clínica en el medio oral, particularmente bajo condiciones en las cuales la adhesión fallaría como consecuencia de una degradación, como sería por ejemplo el ciclado mecánico, ciclado térmico, almacenamiento en agua y otras soluciones (16-22).

\section{Degradación mecánica de la interfase adhesiva}

Los dientes están sujetos continuamente a un estrés durante la masticación, la deglución y los hábitos parafuncionales. El ciclado mecánico, que simula la carga oclusal ejercida por los dientes, podría acelerar el deterioro de la interfase entre la dentina y la restauración $(15,16,18,23)$. Las cargas verticales producidas por una bola de comida entre dientes opuestos se distribuyen sobre toda la cara oclusal, y el estrés es propagado a través de la superficie, lo 
cual puede representar un desafío a la durabilidad a largo plazo de los adhesivos en dentina.

En los últimos años se han desarrollado unos sistemas simuladores de la cavidad oral para ocasionar un estrés mecánico in vitro y evaluar el efecto del ciclado mecánico en las fuerzas de adhesión de adhesivos dentinarios autograbadores y convencionales.

Se ha demostrado que la combinación del simulador de la masticación (cicladora mecánica) en combinación con el test de microtensión pueden proporcionar magníficos resultados in vitro para la evaluación de la durabilidad de la adhesión dentinaria (19).

\section{Método de medida de la eficacia adhesiva en reconstrucciones intracoronales (test de microtensión) (fig. 1)}

Las pruebas de fuerzas de adhesión son las más usadas para cuantificar la eficacia adhesiva de diferentes sistemas. La base de este método es que la adhesión más fuerte entre el diente y el biomaterial, resistirá mejor el estrés impuesto por el sistema y la función oral (24).

A lo largo del tiempo, se han desarrollado diversos tests de fuerzas de adhesión (24). La fuerza producida en los sistemas adhesivos dentinarios se ha eva- luado tradicionalmente usando el test de resistencia al cizallamiento o shear bond strength, el cual, resulta útil para probar materiales que fallan ante valores comprendidos entre 18-20 MPa, o menos (25). Sin embargo, en valores que exceden la citada cifra, a menudo no permiten diferenciar entre la fuerza del adhesivo y la fuerza cohesiva del composite o la dentina (25). Además, debido a que la evaluación exacta de un material adhesivo se determina mejor cuando el fallo ocurre en el propio material y no implica la dentina o el composite y, a que la mejora de los adhesivos dentinarios aumenta con el paso del tiempo, apareció la necesidad de obtener un método mejor y más eficaz. De esta manera, se creó el test de microtensión, que hoy en día es el más usado. La técnica de microtensión para evaluar la resistencia adhesiva introducida por Sano y cols. (26), se trata de una técnica muy laboriosa, pero presenta múltiples ventajas: 1) con ella se pueden medir grandes fuerzas de adhesión; 2) permite testar la adhesión en áreas muy pequeñas y en diferentes regiones y; 3) es capaz de obtener de una sola pieza múltiples especímenes.

Para realizar el test de microtensión en dentina coronal (fig. 1), el espécimen se debe seccionar perpendicularmente a su eje mayor. Las superficies se pulen con una lija de papel de 180 grit bajo una continua corriente de agua hasta exponer una superficie de dentina (fig. 2) y haber removido el esmalte remanente, obteniendo una superficie totalmente plana para realizar la adhesión. Después de preparar las

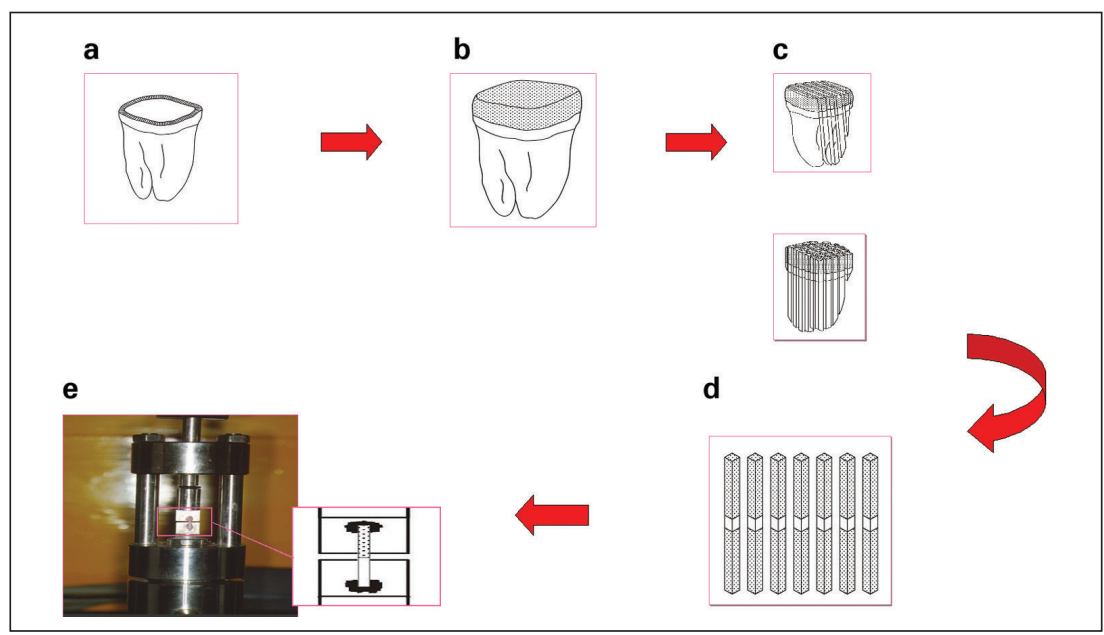

Fig. 1. Esquema del método seguido en el test de microtensión para dentina media coronal sana. a) Los especimenes se seccionan transversalmente; b) tras ser aplicados los adhesivos siguiendo las instrucciones del fabricante, se realiza una corona de composite de $7 \mathrm{~mm}$; c) Después de ser almacenados 24 horas, los especímenes se seccionan en sus ejes " $x$ " $e$ "y"; d) para obtener barritas de un área aproximada de $1 \mathrm{~mm}^{2}$; e) Entonces cada barrita se somete al test de microtensión. 


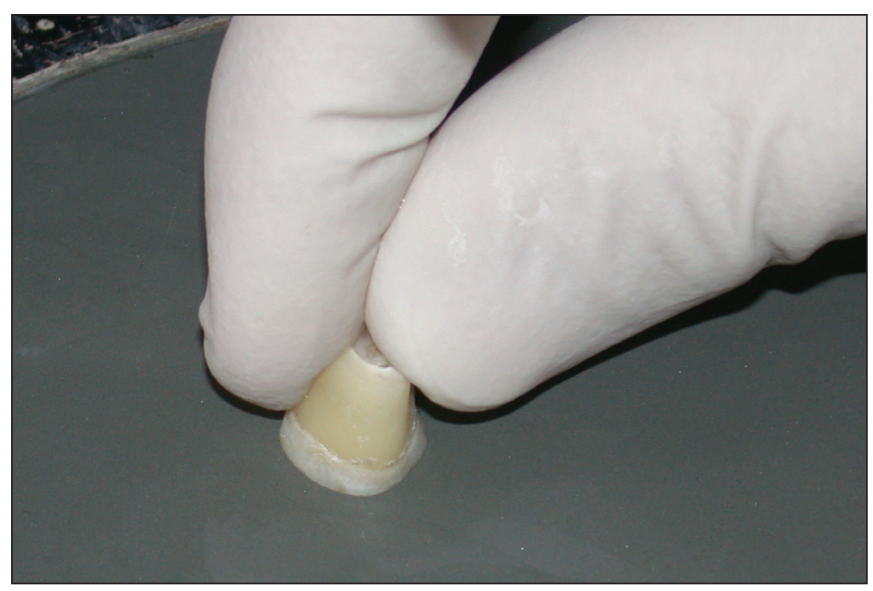

Fig. 2. Fotografía de los especimenes puliéndose en una pulidora mecánica con discos de 180 grit.

superficies, se aplican los agentes adhesivos. Se construye una corona de resina de $7 \mathrm{~mm}$ de altura construida incrementalmente con un composite. Cada capa de composite se fotopolimeriza durante 40 segundos con una unidad de luz, la cual, se debe monitorear con un radiómetro a una temperatura de $21^{\circ}$ y relativa humedad de $61 \%$. Después se deben almacenar los especímenes durante el tiempo requerido, dependiendo del objetivo del estudio, en agua destilada a $37^{\circ} \mathrm{C}$. Se seccionan las piezas a nivel del tercio medio de la raíz y se obturan a retro con un adhesivo y composite, después de haber removido el tejido pulpar. Entonces los especímenes se seccionan verticalmente en intervalos de $1 \mathrm{~mm}$ de espesor, en ambas direcciones "x" e "y" (fig. 3)

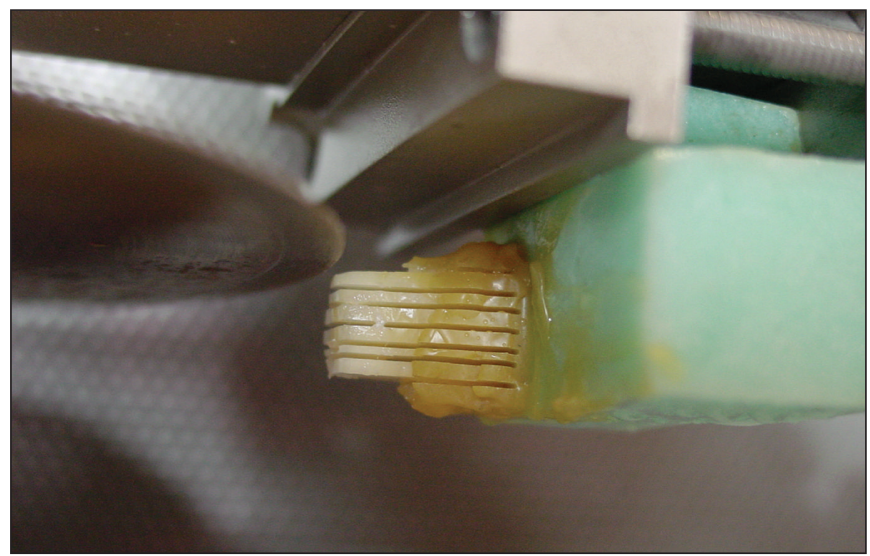

Fig. 3. Imagen de los especimenes seccionándose en una cortadora mecánica para obtener las barritas. usando una máquina de corte siguiendo el método propuesto por Sano y cols. (27). El proceso crea unas barritas con área de aproximadamente $1 \mathrm{~mm}^{2}$ (por ejemplo, $1 \times 1 \mathrm{~mm}$ ). Los especímenes se llevan a una máquina Instron y se pegan a un aparato Bencor Multi-T con un adhesivo de cianocrilato y se somete a una fuerza de tensión en una máquina Instron con una aceleración de $0,5 \mathrm{~mm} / \mathrm{min}$ hasta el fallo (fig. 4). Una vez que se ha removido de las mordazas las barritas fracturadas, se localiza el tipo de fallo con un microscopio electrónico. Clasificándolo según el lugar de la fractura en fallo adhesivo, mixto o cohesivo (fig. 5).

\section{Ciclado mecánico (fig. 6)}

Es difícil, si no imposible, simular exactamente con la cicladora mecánica la fuerza oclusal debido a la variación en la edad, el sexo, el tipo de diente y alimento (18). Anderson y cols. (28) registraron cargas

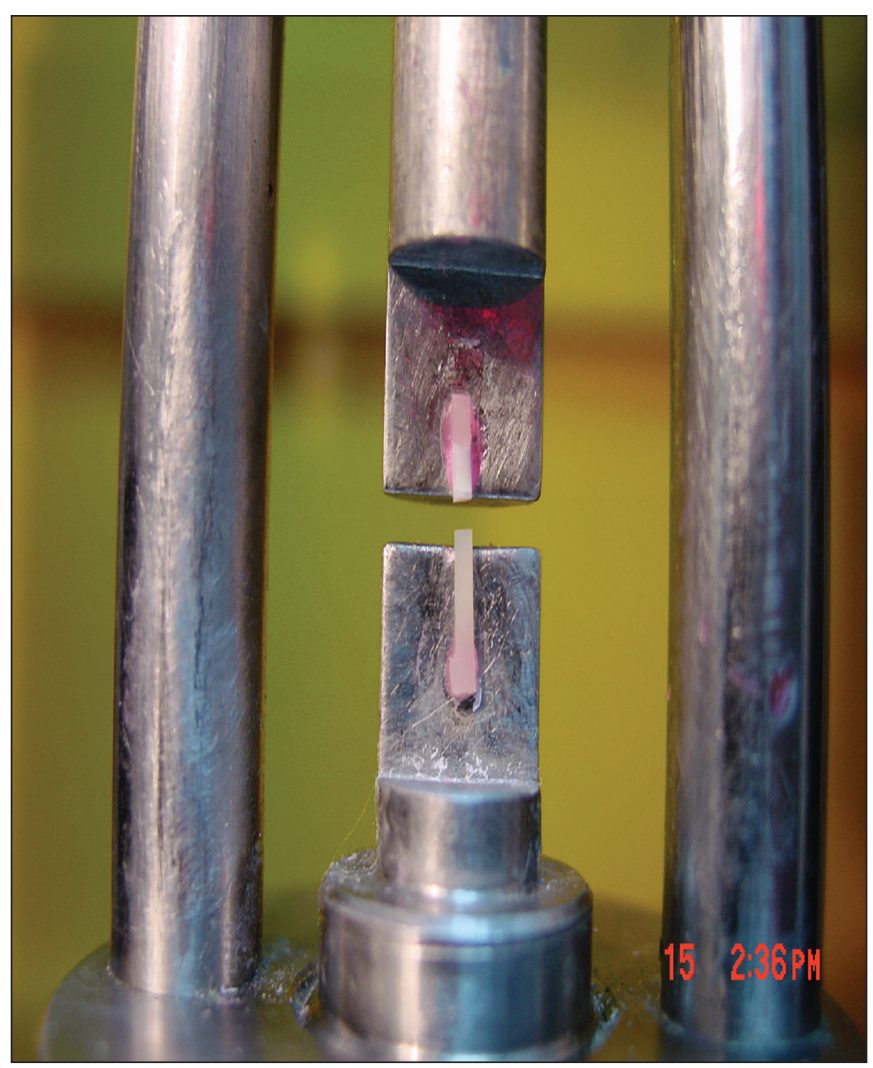

Fig. 4. Barrita de aproximadamente $1 \mathrm{~mm}^{2}$ sometida al test de microtensión una vez fracturada. 


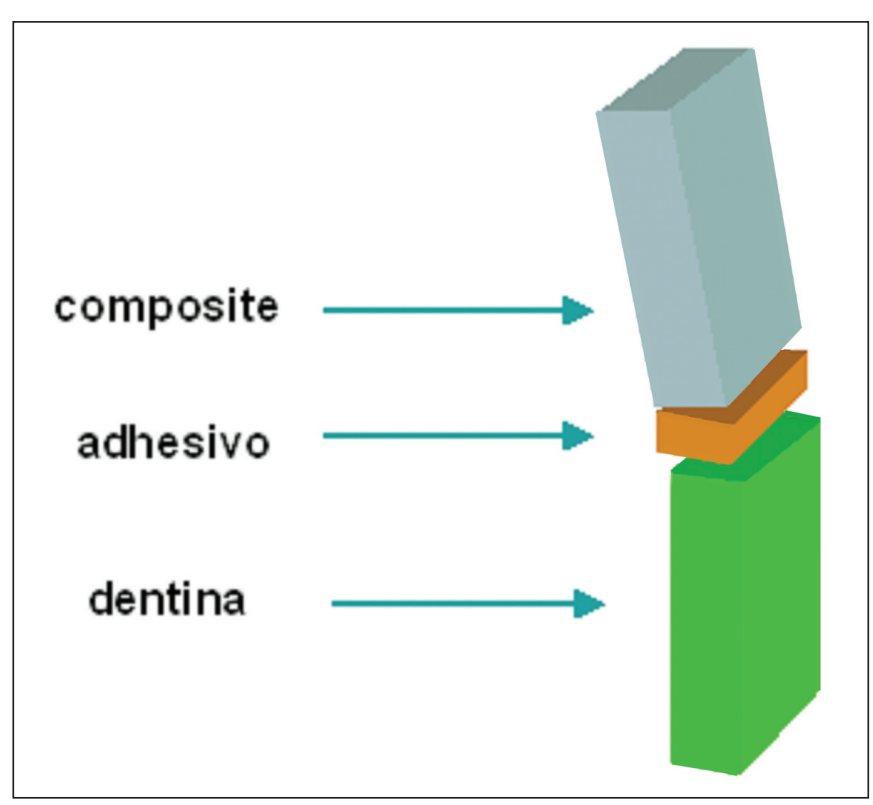

Fig. 5. Esquema del tipo de fallo observado una vez que se ha removido la barrita fracturada de las mordazas. Si la fractura se ha producido en el composite o en la dentina se considera un fallo cohesivo. Si la fractura ha sido en el adhesivo pero con restos de resina, entonces es mixto. Y si por lo contrario es una fractura limpia, se considera un fallo adhesivo.

axiales desde 70 a 150 Newton durante la masticación y la deglución, sin embargo, estas fuerzas se producían en condiciones normales. De este modo, para simular la fuerza sometida sobre un diente se suele usar una fuerza de $90 \mathrm{~N}$ que está en la media (29). Pero puede ser que las fuerzas producidas en la cavidad oral sean mayores debido a anomalías como el bruximo, traumatismo oclusal, etc. Entonces, la carga utilizada se aumenta a $250 \mathrm{~N}$ con la intención de simular dichas fuerzas anómalas (23). El número de ciclos varía entre los diferentes estudios, desde 1.000 a 8.000 , llegando algunos incluso a $50.000(16,19,23,29)$. Unos estudios recientes realizados usando diferentes números de ciclos y diferentes cargas cíclicas (30) han demostrado que la fuerza con la que es golpeado el espécimen no influye en las fuerzas de adhesión, sin embargo, los especímenes que fueron sometidos a 50.000 ciclos experimentaron una mayor disminución de los valores de adhesión que aquellos expuestos a 5.000 ciclos (30).

La formación de la capa híbrida es un importante factor para crear una adhesión óptima entre la resina del adhesivo y la dentina $(3,4,8)$. Se ha demostrado que la formación de una capa híbrida a lo largo de la interfase de toda la restauración es lo más importante para obtener una adhesión resina-dentina duradera (6). Se piensa que las deformaciones de la restauración tienen lugar debido a la carga cíclica, la cual puede causar la creación de microseparaciones entre el suelo de la cavidad y el adhesivo o deformaciones plásticas de la interfase adhesiva (19). El estrés causado por la carga cíclica puede acelerar la degradación o alteración del sustrato periférico de la capa híbrida adhesiva (19), sobre todo en la interfase entre el adhesivo y la parte más alta de la capa híbrida. Aunque en algunas áreas, las fracturas ocurren en el fondo de la capa híbrida (29).

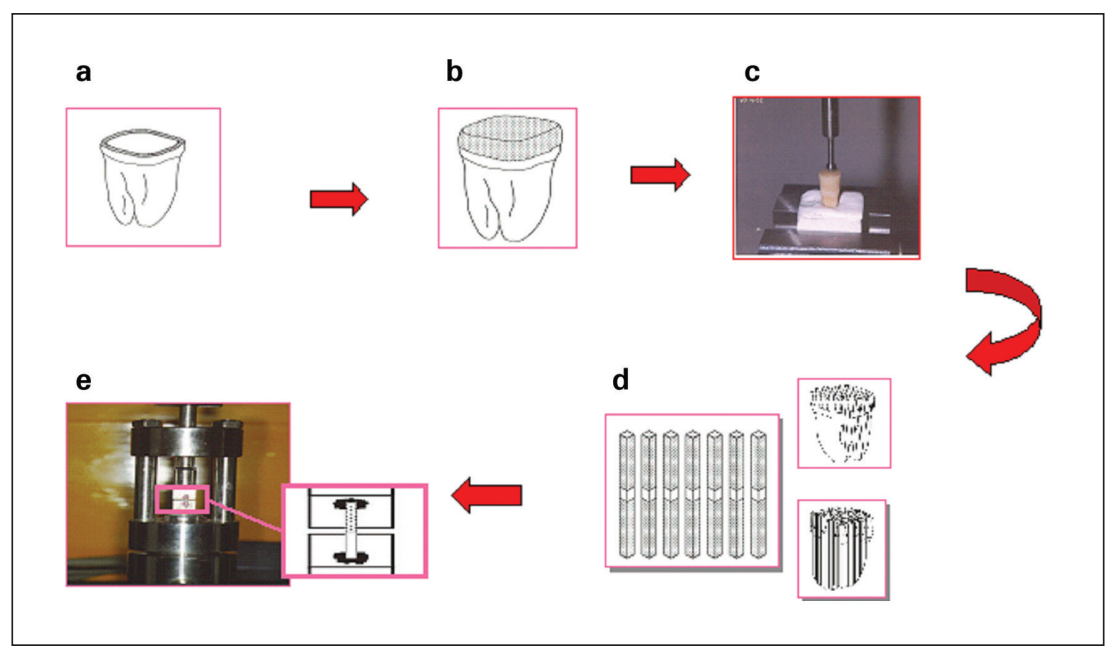

Fig. 6. Esquema del método seguido en los especimenes sometidos a un ciclado mecánico seguidos del test de microtensión para dentina media coronal sana. a) Los especimenes se seccionan transversalmente, b) tras aplicarse los adhesivos siguiendo las instrucciones del fabricante, se realiza una corona de composite de $7 \mathrm{~mm}$. c) Después de almacenarlos durante 24 horas, los especimenes se montan en unos cubos de resina y son ciclados en la cicladora mecánica d) A continuación, los especimenes se seccionan en sus ejes "x" e "y" para obtener barritas de un área aproximada de $1 \mathrm{~mm}^{2}$. e) Entonces cada barrita se someta al test de microtensión. 
Los estudios más recientes han demostrado que los valores de adhesión resina-dentina disminuyen tras el ciclado mecánico $(23,29,30)$. Además, si la dentina se graba con ácido ortofosfórico, es decir, se usan sistemas de grabado total, los adhesivos que tienen alcohol como solvente se comportan mejor que aquellos que contienen acetona (29). Los adhesivos autograbadores de un solo paso muestran las fuerzas de adhesión más bajas y menos duraderas, mientras que los adhesivos autograbadores de dos pasos obtienen los valores de adhesión más altos (31).

Para realizar un test de ciclado mecánico con la intención de evaluar la durabilidad de la interfase adhesiva resina-dentina, los especímenes se deben preparar de la misma manera que si fuesen a ser sometidos a un test de microtensión (comentado anteriormente). De esta manera, una vez que se han aplicado los adhesivos, realizado la corona con composite y almacenados en la estufa a $37^{\circ}$ durante el tiempo que lo estipulaba el protocolo, los especímenes deben ser sometidos a un ciclado mecánico (con un determinado número de ciclos (veces que se golpea el diente), una fuerza determinada y una frecuencia (número de golpes por segundo) antes de continuar con el protocolo del test de microtensión y ser seccionados en barritas para que sean testados en la máquina Instron. Para el test del ciclado mecánico, los dientes se deben montar en unos cubos de resina acrílica Idopol Trays (Unidesa, Madrid) (fig. 7 a, b, c, d) y ciclados con la fuerza centrada en la mitad de la corona de resina (fig. 7 e, f), aplicado por una bola de $5.0 \mathrm{~mm}$ de diámetro rígidamente unida a el golpeador de la máquina cicladora.

\section{Degradación química de la interfase adhesiva}

La dentina es un substrato formado por compuestos orgánicos e inorgánicos. La fase orgánica está representada principalmente por una estructura fibrosa de colágeno que puede ser degradada por enzimas pro-

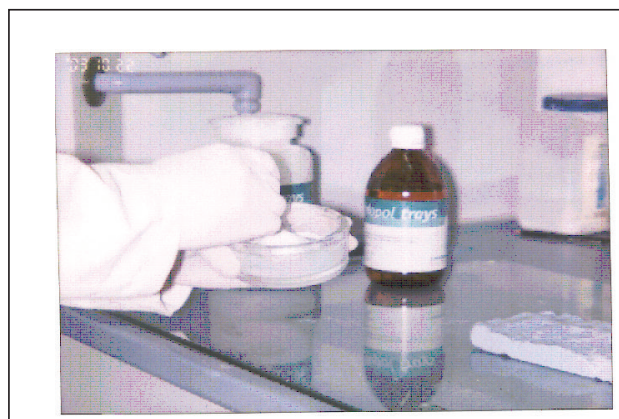

a

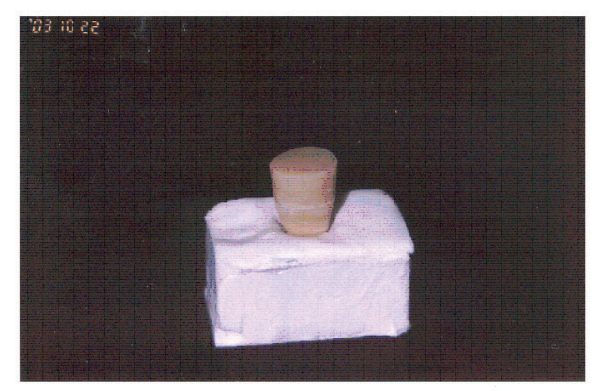

d

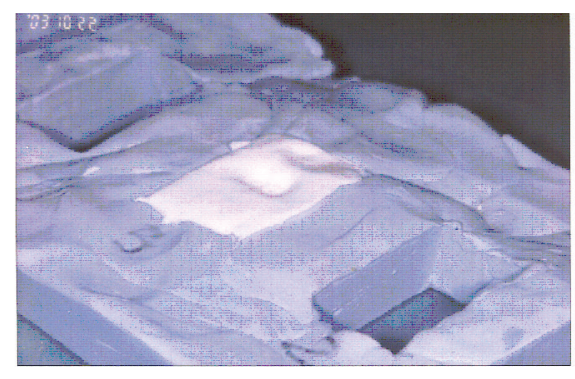

b

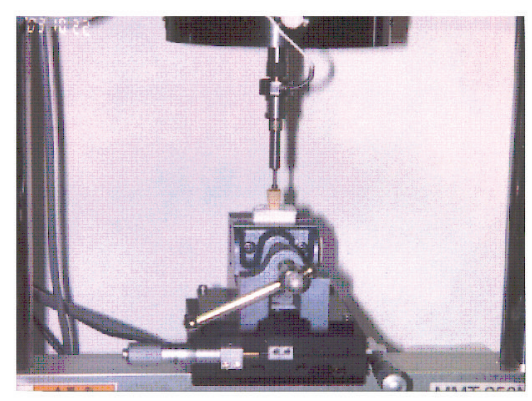

e

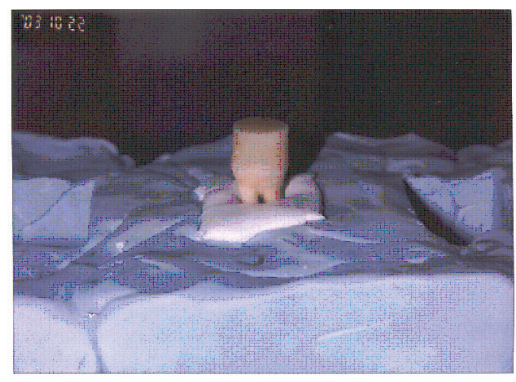

C

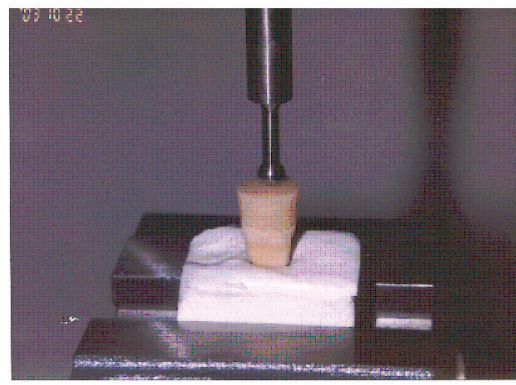

f

Fig. 7. Esquema de la preparación del espécimen para ser testado en el test de ciclado mecánico. (a) Preparación de la resina Idopol Trays. (b) La resina se introduce en los moldes de silicona. (c) Dos tercios de la raíz del espécimen se sumergen en la resina. (d) Al endurecer, ya está preparado para introducirse en la cicladora mecánica y ciclarse en seco (e) con una fuerza constante en cada golpe durante un número de ciclos determinado (f). 
teolíticas $(32,33)$. Es posible que tales enzimas afecten la durabilidad de la adhesión a dentina si los componentes orgánicos, tales como el colágeno, se dejan expuesto después de la infiltración de resina. El hipoclorito de sodio ( $\mathrm{NaOCl}$ ) se ha utilizado como un sustituto de enzimas proteolíticas $(20,34)$. La solución de $\mathrm{NaOCl}$ tiene un efecto proteolítico no-específico que elimina efectivamente los componentes orgánicos de dientes que han sido desmineralizados pero han quedado sin ser infiltrados por el primer (20).

Se han propuesto diversas pruebas in vitro con el objetivo de producir un envejecimiento acelerado de la interfase formada por los adhesivos dentinarios, reduciendo el tamaño de los especímenes a barritas y sumergiendo éstas en hipoclorito de sodio al $10 \%$ acuoso $\left(\mathrm{NaOCl}_{\mathrm{aq}}\right)$ por un periodo de tiempo experimental corto, con la intención de determinar la habilidad de los monómeros de resina de proteger la matriz de colágeno de la actividad del $\mathrm{NaOCl}_{\mathrm{aq}}(20$, 35). De esta manera, la evidencia proporcionada por unos previos estudios realizados in vivo e in vitro de la hidrólisis en los adhesivos haría del $\mathrm{NaOCl}_{\mathrm{aq}}$ un buen medio de prueba para analizar la durabilidad de los adhesivos (20).

Los estudios de envejecimiento in vitro a largo plazo que usaron agua como medio de almacenamiento han demostrado que producen una disminución de las fuerzas de adhesión creadas en la interfase resina-dentina $(17,22)$. Se ha demostrado que el $\mathrm{NaOCl}$ podría realizar el mismo efecto obtenido con meses de almacenamiento en agua, por lo que supondría un método de envejecimiento in vitro mucho más rápido $(20,35)$. Tras la inmersión en $\mathrm{NaOCl}_{\mathrm{aq}}$, la interfaz resina-dentina puede degradarse químicamente. La disminución en las fuerzas de adhesión de todos los sistemas adhesivos es el resultado de un proceso de solubilización de la resina y de las fibras de colágeno desprotegidas en la dentina descalcificada (40).

Recientes estudios realizados in vivo demostraron que las fuerzas de adhesión de los adhesivos de grabado total de tres pasos pueden reducirse entre un 50-65\% después de 2-3 años (32). Cuando se usan adhesivos de grabado total de dos pasos, las fuerzas de adhesión disminuyen un $65 \%$ en un año y un $77 \%$ cuando se usan adhesivos autograbadores de dos pasos (36). Sin embargo, algunos estudios in vivo basados en el almacenamiento con agua dieron una disminución menor de las fuerzas de adhesión, entre 23-55\% (37, 38, 39), incluso después de ser expuesta directamente la interfaz resina-dentina con el agua durante seis años (37). La disminución de las fuerzas de adhesión obtenidas tras la inmersión en $\mathrm{NaOCl}_{\text {aq }}$ fueron entre $65 \%-77 \%(20,35)$, es decir, similares a la disminución de las fuerzas de adhesión obtenidas en los estudios realizados in vivo $(32,36)$. Por todo esto deducimos que el método de envejecimiento basado en la inmersión en $\mathrm{NaOCl}_{\mathrm{aq}}$ durante un corto periodo de tiempo obtiene datos más reales que los estudios in vitro basados en el almacenamiento de los especímenes en agua (40). Los estudios in vivo encontraron que el aspecto microestructural de las superficies despegadas sugieren que el colágeno expuesto fue hidrolizado por enzimas proteolíticas (41), las cuales, pueden estar relacionadas con leucocitos, glándulas salivares o bacterias de la placa (35).

Para realizar el test de microtensión junto a una degradación en $\mathrm{NaOCl}_{\mathrm{aq}}$ (fig. 8) se debe realizar el mismo protocolo expuesto anteriormente en el apartado del test de microtensión, con la diferencia de almacenar en $\mathrm{NaOCl}_{\mathrm{aq}}$ durante 5 horas (fig. 9) en una

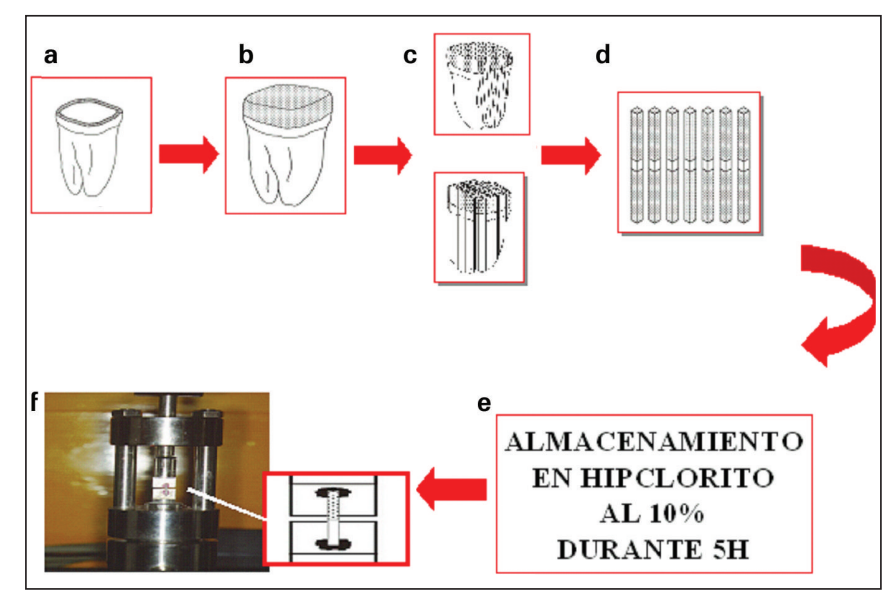

Fig. 8. Esquema del método seguido en los especimenes envejecidos con el test de inmersión en $\mathrm{NaOCl}_{\text {aq }}$ al $10 \%$ durante 5 horas seguidos del test de microtensión. a) Los especimenes se seccionan transversalmente, b) tras ser aplicados los adhesivos siguiendo las instrucciones del fabricante, se realiza una corona de composite de $7 \mathrm{~mm}$. c) Los especimenes se seccionan en sus ejes "x" e "y" para obtener barritas de un área aproximada de $1 \mathrm{~mm}^{2}$. d) Las barritas se almacenan durante 5 horas en hipoclorito de sodio al $10 \%$ en una estufa a $37^{\circ} \mathrm{C}$. e) Entonces las barritas se enjuagan en agua y se llevan al test de microtensión. 


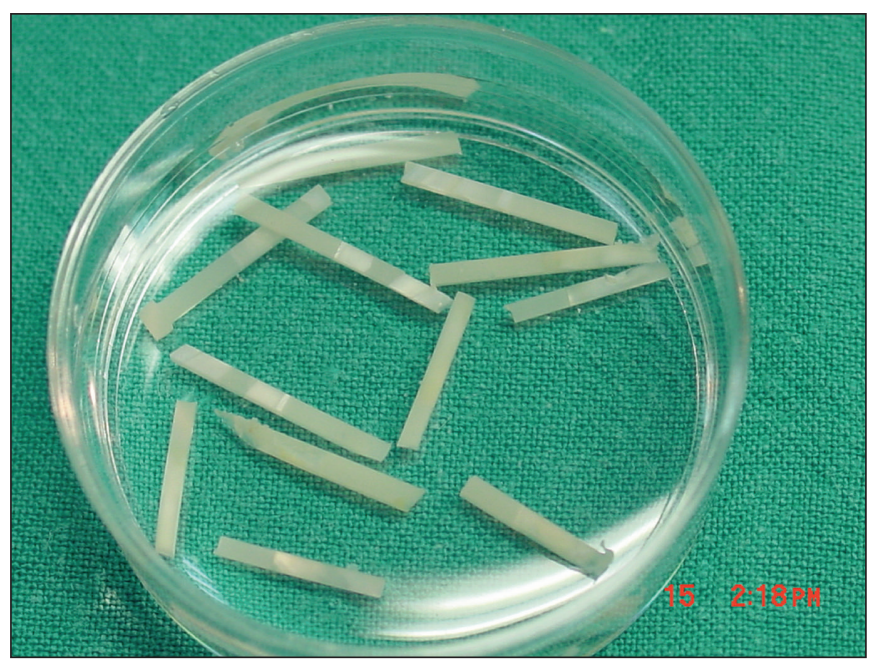

Figura 9: Barritas con área de aproximadamente $1 \mathrm{~mm}^{2}$ introducidas en un recipiente que contiene $\mathrm{NaOCl}_{\mathrm{aq}}$ para ser almacenadas durante 5 horas en una estufa a $37^{\circ} \mathrm{C}$.

estufa a $37^{\circ} \mathrm{C}$, las barritas obtenidas al seccionar el espécimen verticalmente en intervalos de $1 \mathrm{~mm}^{2}$ de espesor (fig. 3). Una vez transcurrido ese tiempo se deben enjuagar con abundante agua cada una de las barritas, y entonces testarlas en la máquina Instron.

\section{CONCLUSIÓN}

El uso combinado del ciclado mecánico con el test de microtensión permite la evaluación in vitro de la durabilidad de la adhesión resina-dentina bajo unas condiciones clínicamente más relevantes de las que son usadas normalmente en la técnica de fuerzas de adhesión estáticas. El método de envejecimiento basado en la inmersión en $\mathrm{NaOCl}_{\mathrm{aq}}$ durante un corto periodo de tiempo obtiene datos más reales que los estudios in vitro basados en el almacenamiento de los especímenes en agua.

\section{BIBLIOGRAFÍA}

1. Sainz de Robles FC. Ensayo de un diccionario español de sinónimos y antónimos, $8^{\mathrm{a}}$ ed. Gredos, Madrid, 1967.

2. Marshall GW, Yücel N, Balooch M, Kinney JH, Habelitz S, Marshall SJ. Sodium hypochlorite alterations of dentin and dentin collagen. Surface Sci 2001;491:444-55.

3. Toledano M, Osorio R, Leonardi de G, RosalesLeal JI, Ceballos L, Cabrerizo-Vilchez MA. Influence of self-etching primer on the resin adhesion to enamel and dentin. Am J Dent 2001; 14:205-10.

4. Osorio R, Toledano M, De Leonardi G, Tay F. Microleakage and interfacial morphology of selfetching adhesives in class $\mathrm{V}$ resin composite restorations. J Biomed Mat Res Part B: Appl Biomat 2003;66B:399-409.

5. De Munck J, Van Meerbeek B, Satoshi I, Vargas M, Yoshida Y, Armstrong S, et al. Microtensile bond strengths of one and two step self-etch adhesives to bur-cut enamel and dentin. Am J Dent 2003;16:414-20.

6. Nakabayashi N, Kojima K, Masuhara E. The promotion of adhesion by the infiltration of monomers into tooth substrates. J Biomed Mater Res 1982;16:265-73.

7. Nakabayashi N, Nakamura M, Yasuda N. Hybrid layer as a dentin-bonding mechanism. J Esthetic Dent 1991;3:133-8.

8. Van Meerbeek B, Inokoshi S, Braen M, Lambrechts P, Vanherle G. Morphological aspects of the resin-dentin interdifussion zone with different dentin adhesive systems. J Dent Res 1992;71:1530-40.

9. Gwinnet AJ. Quantitative contribution of resin infiltration/hybridization to dentin bonding. Am J Dent 1993;6:7-9.

10. Toledano M, Osorio R, Ceballos L, Fuentes MV, Fernandes CAO, Tay FR, et al. Microtensile bond strength of several different adhesive systems to different dentin depths. Am J Dent 2003; 16 : 292-8.

11. Mjör IA, Nordahl I. The density and branching of dentinal tubules in human teeth. Arch Oral Biol 1996; 8:541-6. 
12. Toledano M, Osorio R, Sánchez F, Osorio E. Arte y Ciencia de los Materiales Dentales. 2003, Ediciones Avances Medico-Dentales, S.L. Madrid.

13. Fritz UB, Finger WJ. Bonding efficiency of single-bottle enamel/dentin adhesives. Am J Dent 1999;12:277-82.

14. Toledano M, Osorio R, Osorio E, Prai C, Carvalho R. Microhardness of acid-treated and resin infiltrated human dentine. J Dent 2004;33:34954.

15. Osorio R, Toledano M, Osorio E, Aguilera FS, Tay FR. Effect of load cycling and in vitro degradation on resin-dentin bonds using a selfetching primer. J Biomed Mat Res Part A: Appl Biomat 2005;72A:399-408.

16. Abdalla A, Davidson C. Effect of mechanical load cycling on the marginal integrity of adhesive class I resin composite restorations. J Dent 1996; 24: 87-90.

17. Kato G, Nakabayashi N. The durability of adhesion to phosphoric acid etched, wet dentin substrates. Dent Mater 1998;14:347-52.

18. Nikaido T, Kunzelmann $\mathrm{KH}$, Chen $\mathrm{H}$, Ogata $M$, Harada N, Yamaguchi S, et al. Evaluation of thermal cycling and mechnical loading on bond strength of a self-etching primer system to dentin. Dent Mater 2002;18:269-75.

19. Nikaido T, Kunzelmann KH, Ogata M, Harada N, Yamaguchi S, Cox CF, et al. The in vitro dentin bond strength of two adhesive systems in Class I cavities of human molars. J Adhes Dent 2002;4: 31-9.

20. Yamauti $M$, Hashimoto $M$, Sano $H$, Ohno $H$, Carvalho R.M, Kaga M, et al. Degradation of resindentin bonds using $\mathrm{NaOCl}$ storage. Dent Mater 2003;19:399-405.

21. Osorio R, Toledano M, Osorio E, Tay FR. Longevity of bonds made by composite and polyacidmodified resins to dentin using a dual-cured adhesive system. Am J Dent 2005;18:19-22.
22. Burrow MF, Satoh M, Tagami J. Dentin bod durability after three years using a dentin bonding agent with and without priming. Dent Mater 1996; 12:302-7.

23. Albaladejo A, Osorio R, Aguilera F.S, Tay F.R, Toledano M. Effect of mechanical loading on bond strength of self-priming and self-etching adhesive systems to dentin. J Dent Res 2005; 84(Sp Iss A):2978.

24. Pashley DH, Sano H, Ciucchi B, Yoshiyama M, Carvalho RM. Adhesion testing of dentin bonding agents: A review. Dent Mater 1995;11: 117-25.

25. Chappell RP, Cobb CM, Spencer P, Eick JD. Dentinal tubule anastomosis: a potencial factor in adhesive bonding?. J Prosthet Dent 1997;72: 183-8.

26. Sano H, Shono T, Sonoda H. Relationship between surface area for adhesion and tensile bond strength- evaluation of a micro-tensile bond test. Dent Mater 1994;10:236-40.

27. Sano H, Yoshikawa T, Pereira PNR, Kanemura N, Morigami M, Tagami J, et al. Long-term durability of dentin bonds made with a self-etching primer. J Dent Res 1999;78:906-11.

28. Anderson DJ. Measurement of stress in mastication II. J Dent Res 1956;35(5):671-3.

29. Toledano M, Osorio R, Albaladejo A, Aguilera FS, Tay FR, Ferrari M. Effect of cyclic loading on microtensile bond strengths of total-etch and selfetch adhesives. Oper Dent 2006;31:25-32.

30. Albaladejo A, Toledano M, Aguilera FS, Osorio R. ABF Antibacterial Adhesive System: In Vitro Durability Evaluation of the Dentin Bond Strengths. Annual Meeting of the Academy of Dental Materials. Geneva, 2004;18:150.

31. Albaladejo A, Osorio R, Toledano M. In Vitro Challenging of Experimental Self-Priming Adhesive Systems. J Dent Res 2006; 85(Sp Iss A):1309. 
32. Hashimoto M, Ohno H, Kaga M, Endo K, Sano h, Oguchi $H$. In vivo de gradation of resin-dentin bonds in humans over 1 to 3 years. J Dent Res 2000; 79(6): 1385-91.

33. Hashimoto M, Ohno H, Kaga M, Endo K, Sano $\mathrm{H}$, Oguchi $\mathrm{H}$. Resin-tooth adhesive interfaces after long-term function in humans. Am J Dent 2001; 14: 211-5.

34. Spencer P, Swafford JR. Unprotected protein at the dentin-adhesive interface. Quintessence Int 1999; 30:501-7.

35. Yoshida E, Hashimoto M, Hori M, Kaga M, Sano $\mathrm{H}$, Oguchi H. Deproteinizing effects on resin-tooth bond structures. J Biomed Mater Res Part B: Appl Biomat 2004; 68B: 29-35.

36. Koshiro K, Inoue S, Tanaka T, Koase K, Fujita M, Hashimoto $M$, et al. In vivo degradation of resindentin bonds produced by self-etch vs. a totaletch adhesive system. Eur J Oral Sci 2004; 112: 368-75.

37. Frankenberger R, Strobel W O, Lohbauer U, Krämer N, Petschelt A. the effect of six years of water storage on resin composite bonding to human dentin. J Biomed Mater Res Part B:Appl Biomat 2004; 69B: 25-32.

38. Okuda M, Pereira PNR, Nakajima M, Tagami J. Relationship between nanoleakage and log-term durability of dentin bonds. Oper Dent 2001;26: 482-90.

39. Reis A, Loguercio AD, Carvalho RM, Grande RHM. Durability of resin dentin interfaces: effects of surface moisture and adhesive solvent component. Dent Mat 2004; 20: 669-76.

40. Toledano M, Osorio R, Albaladejo A, Aguilera FS, Osorio E. Differential effect of in vitro degradation on resin-dentin bonds produced by self-etch vs. total-etch adhesives. J Biomed Mat Res Part A: Appl Biomat 2006; 77(1): 128-35.

\section{CORRESPONDENCIA}

Alberto Albaladejo Martínez

Parque del Genil, Edificio Guadalquivir, 5º-B-2 18004 Granada.

Email: albertoalbaladejo@hotmail.com 